\title{
ERK-dependent mTOR pathway is involved in berberine-induced autophagy in hepatic steatosis
}

Qin He1, Dan Mei1, Sha Sha1, Shanshan Fan1, Lin Wang² and Ming Dong1

'Department of Endocrine and Metabolism, Qilu Hospital of Shandong University, Shandong University, Ji'nan, China 2Department of Endocrine and Metabolism, Jiaxiang People's Hospital, Ji'ning, China

Correspondence should be addressed to M Dong

Email

dr_dongming@126.com

The authors and journal apologise for an error in the above paper, which appeared in volume 57 part 4, pages 251-260. The error relates to the Funding section on page 259.

The original paper stated:

'This work was supported by the Medical and Health Science \& Technology development plan of Shandong Province (no. 2014WS0136), and the Fundamental Research Funds of Qilu Hospital of Shandong University (no. 26010175616016).'

This should have stated:

'This work was supported by the Medical and Health Science \& Technology development plan of Shandong Province (no. 2014WS0136) and the Fundamental Research Funds of Qilu Hospital of Shandong University (no.2015QLMS15).' 